\title{
Letter to editor: the burden of covid-19 in neuro-oncological patients
}

\author{
Simonetti Giorgia ${ }^{1}$ (I) $\cdot$ Gaviani Paola ${ }^{1} \cdot$ Silvani Antonio ${ }^{1}$
}

Received: 10 April 2020 / Accepted: 25 April 2020 / Published online: 18 May 2020

(c) Springer Science+Business Media, LLC, part of Springer Nature 2020

\section{To editor}

Covid-19 is acting as an epochal event, not only for the health impact, with an amount of 1.631 .310 people infected [1], but also for political, social and economic consequences. The fast spreading of the coronavirus worldwide, forced hospitals to review working priorities.

Modern health care systems are based on the theory of "patient-centered" care, but coronavirus epidemic forced a change toward a "community-centered" care perspective.

In Italy, and in particular in the North, a massive reorganization of the whole public health system occurred at breathtaking speed, with the creation of COVID-free and COVID + hospitals and dedicated neurointensive care units. Draconian strategies were imposed nationwide to fight the spread of the COVID-19 infection and also to protect frail patients such as oncological and elderly, subject to higher risk of health complications. Evidence suggested that patients with a history of cancer had a higher incidence of severe complications, compared with other patients who did not have cancer. Such complications may include, intensive care assistance, mechanical ventilation or death [2].

In light of this, many recommendations invited physicians to consider the possibility to postpone non-urgent visits and anticancer therapies in order to decrease the interaction between patients and health care system, a setting strongly correlated to a higher risk of getting an infection. In this scenario, neuro-oncological patients seem to have some important implications.

Malignant brain tumors are relatively rare cancer with high degree of lethality and inevitable traumatic impact on patient's life and caregivers. Due to the rarity and the complexity of the disease, the management of neuro-oncological patients is hyper-specialistic and generally based on hub

Simonetti Giorgia

giorgia.simonetti@istituto-besta.it

1 Neuro-Oncology Unit, Department of Clinical Neurosciences, Fondazione IRCCS Istituto Neurologico

Carlo Besta, Via Celoria, 11, 20133 Milan, Italy hospitals which offer a full array of services. On the other hands, spoke hospitals are lacking and patients have to move on territory when needing more intensive services.

Currently, nationwide regulations on travel and quarantine, imposed countries lockdown.

Not rarely, in order to contain the exceptional lack of healthcare personnel and avoid national health system collapse, physicians and nurses afferent to neuro-oncology field, were siphoned off to various operational units and divisions to treat acutely ill COVID19 non-neuro-oncological patients.

For neuro-oncological patients in particular, this situation seems to further complicate their management; although life-saving treatments are indeed guaranteed, access to scheduled or unscheduled visits for evaluations and prescriptions became even more difficult, if not impossible outside the region, and in any case discouraged.

In few weeks, we have witnessed growing anxiety, fear of abandonment, discouragement both in patients and caregivers, due to the sudden changed relationship with neurooncologist and difficulties to trust in home health care.

COVID-19 is causing a more silence but very dangerous emergency on frail oncological population.

Serious psychological sequelae could develop in patients already downhearted by devastating diagnosis with poor chances for survival [3]. Uncertainty for the future, fear for loved ones and for themselves, radical upheavals of daily habits, are only the most common conditions that can aggravate anxiety situations. Moreover, interrupted support systems and social isolation can leave vulnerable patients particularly sensitive to acute stress reactions. For these reasons, the uncontrolled pandemic could expose neurooncological patients to an overbearing emotional reaction, because of their higher susceptibility to stress compared to the general population.

In this challenging scenario, the first concern is to continue the support of neuro-oncological patients and their needs. It is essential to preserve communication between clinicians, patients and caregivers. Moreover, efforts should be made in order to guarantee the quality of care across the country and attempt to avoid sense of abandonment. 
In our Neuro-oncology Division, about $80 \%$ of in-person visits were cut in the last two months. Only $20 \%$ of neurooncological patients followed by our Division continued to access directly to the Hospital, in particular those with complex health conditions or those who needed to start new adjuvant radio or chemotherapy.

The remaining patients, were managed throughout telemedicine, using electronic information and communications technology to provide assistance and medical support.

Where possible, also the dispensing of oral chemotherapies was delegated from the hospital pharmacy to local healthcare services. Digital images, documents, MRIs, blood test results, needed before the start of chemotherapy, were sent to neuro-oncologist for review. Also, real-time interaction between patients and physicians was implemented through audio-visual telecommunication technology.

This was the easiest way to promptly obtain telemedicine service with immediate available devices that allowed to reach patients and self-isolated people at home and to fill gaps of access to local health services, mainly in rural areas.

But the prospective of a prolonged period of social distancing, imposes to develop structured e-Health models in order to sustain the forthcoming health care system revolution.

A reasonable strategy should contemplate the presence of a network between hub and spoke centers, where to take advantages of competences and collaboration of all specialists involved. We believe that the development of technological tools is crucial to enable the implementation of telemedicine.

Dedicated hardware/software systems for data acquisition, communication, processing, storage via mobile devices and web applications should be realized to facilitate bidirectional sharing of health information, guaranteeing patients data security and privacy.

This will ensure reliable and equitable delivery of safe and high-quality care to improve outcomes of brain cancer patients, saving them from traveling long distances. E-Health will be able also to increase patients and caregivers empowerment allowing to self-manage chronic situations [4]. At the same time, the network could provide a tool for healthcare members to identify opportunities for collaboration and to better understand the interaction between telemedicine/telehealth services and health information technology applications at the local, regional and national levels.

Such a digital revolution is not to be intended only as a tool to face this global emergency, but also as a chance for the next future. Digital revolution will help health care system and patients not only today, but also tomorrow.

\section{Compliance with ethical standards}

Conflict of interest The authors declare that they have no conflict of interest.

\section{References}

1. COVID-19 information and resources for JHU in https://hub.jhu. edu/novel-coronavirusinformation. Available at: https://coron avirus.jhu.edu/map.html. Accessed April 10, 2020

2. Liang W, Guan W, Chen R et al (2020) Cancer patients in SARSCoV-2 infection: a nationwide analysis in China. Lancet Oncol 21(3):335-337

3. Stieb S, Fischbeck S, Wagner W et al (2018) High psychosocial burden in relatives of malignant brain tumor patients. Clin Neurol Neurosurg 170:1-6

4. Kondylakis H, Bucur A, Crico C et al (2020) Patient empowerment for cancer patients through a novel ICT infrastructure. J Biomed Inform 101:103342

Publisher's Note Springer Nature remains neutral with regard to jurisdictional claims in published maps and institutional affiliations. 